\title{
Aging-related attention deficits in frequency discrimination amid task-irrelevant stimulus differences
}

\author{
Blas Espinoza-Varas ${ }^{1, *}$, Praveen Jajoria ${ }^{2}$ and Hyunsook Jang ${ }^{3}$ \\ ${ }^{1}$ Department Communication Sciences and Disorders, University Oklahoma Health Sciences Center, \\ Oklahoma City, Oklahoma 73104, USA \\ ${ }^{2}$ Pinnacle Health Rheumatology Associates, 810 Sir Thomas Court, Suite 1, Harrisburg, PA 17109, USA \\ ${ }^{3}$ Division of Speech Pathology and Audiology, Hallym University, Chuncheon, South Korea
}

(Received 25 January 2019, Accepted for publication 30 January 2019)

Keywords: Aging, Selective attention, Irrelevant information, Frequency discrimination, Cognitive deficits PACS number: 43.66.Fe, 43.66.Sr [doi:10.1250/ast.40.225]

\section{Introduction}

Difficulty ignoring irrelevant auditory information (IAI) such as speech babble at a social gathering is a cognitive deficit that aggravates with aging [1], possibly reflecting a pervasive inhibitory deficit [2] that clutters and disrupts the capacity-limited human information processing [3]. Research on this deficit has employed mostly speech stimuli that are ecologically meaningful but fail to reveal the specifics of how task-relevant and task-irrelevant speech information interact with one another at the perceptual level, resulting in failure to ignore the latter [4]. As a result, unveiling basic mechanisms accounting for failure to ignore IAI has remained elusive. To circumvent this limitation, in young and older adults, we are studying the ability to ignore IAI with psychoacoustic discrimination tasks and well-controlled stimuli [5,6]; here, we studied aging effects on the ability to ignore taskirrelevant level or duration differences while participants focused on task-relevant frequency differences. In order to engender task-irrelevant information in frequency-discrimination tasks the stimuli must include systematic (rather than random) and discernible differences along the task-relevant and a task-irrelevant dimension (e.g., level or duration). In addition, the differences along the two dimensions must convey conflicting information so that, to respond correctly, participants must succeed both at attending to the relevant dimension and at ignoring the irrelevant one, enabling them to extricate relevant from the irrelevant information at the perceptual level [1].

In forced-choice frequency discrimination tasks with two observation intervals (2I/2AFC), irrelevant information can be engendered by including irrelevant differences in the signal level, either within trials, between trials, or both $[7,8]$. Compared to fixed-level conditions, random within-trial level differences increase the frequency discrimination thresholds (FDTs) of well-trained young adults by a small but significant amount; i.e., they can ignore irrelevant level differences with modest difficulty. We modified the $2 \mathrm{I} / 2 \mathrm{AFC}$ paradigm to assess aging effects on the ability to ignore irrelevant stimulus differences. The 3-observation interval, 2-alternative forcedchoice (3I/2AFC) task presented a standard (S) tone followed

*e-mail: blas-espinoza-varas@ouhsc.edu by two comparison tones ( $\mathrm{C} 1$ and $\mathrm{C} 2$ ), one of which included a task-relevant frequency difference $\left(\Delta F_{\mathrm{r}}\right)$ relative to the $\mathrm{S}$ frequency. In the absence of task-irrelevant stimulus differences, participants had to report which comparison included $\Delta F_{\mathrm{r}}$. In addition to $\Delta F_{\mathrm{r}}$, in conditions including taskirrelevant differences, both $\mathrm{C} 1$ and $\mathrm{C} 2$ included also a taskirrelevant difference in duration $\left(\Delta T_{\mathrm{i}}\right)$ or in level $\left(\Delta L_{\mathrm{i}}\right)$ relative to $\mathrm{S}$. Thus, both $\mathrm{C} 1$ and $\mathrm{C} 2$ were different from $\mathrm{S}$ in terms of duration or level, but in terms of frequency only one was different from $\mathrm{S}$ (i.e., one comparison conveyed $\Delta F_{\mathrm{r}}$ plus $\Delta T_{\mathrm{i}}$ or $\Delta L_{\mathrm{i}}$ ); responding correctly required ignoring $\Delta L_{\mathrm{i}}$ or $\Delta T_{\mathrm{i}}$ and attending selectively to $\Delta F_{\mathrm{r}}$. Using the $3 \mathrm{I} / 2 \mathrm{AFC}$ task, in a sample of young and one of older adults, we measured FDTs in the absence and in the presence of taskirrelevant level $\left(\Delta L_{\mathrm{i}}\right)$ or duration $\left(\Delta T_{\mathrm{i}}\right)$ differences. Since interactions between pitch and loudness are stronger than between pitch and duration [5,6], we predicted that ignoring $\Delta L_{\mathrm{i}}$ would be much harder than $\Delta T_{\mathrm{i}}$, especially in older adults. Testing this prediction would determine if the older adult's attention deficit is all-inclusive entailing all combinations of task-relevant and task-irrelevant differences, or is specific entailing some combinations but not others; this test would unveil possible mechanisms accounting for failure to ignore irrelevant information.

\section{Method}

\subsection{Equipment}

An Apple computer running LabVIEW-2 software synthesized and gated the pure tones. Analog signals from the D/A converters were low-pass filtered, amplified, attenuated, and fed to earphones (TDH-49). LabVIEW-2 specified the pure tones frequency and duration, and a programmable attenuator controlled the level. Tones were presented monaurally, and participants responded on a computer keyboard in keeping with instructions and feedback displayed on a monitor; the computer controlled all experiment events.

\subsection{Experimental task}

For $1,000-\mathrm{Hz}, 80-\mathrm{ms}, 75-\mathrm{dB}$ SPL target tones, we measured FDTs for positive frequency differences presented either in the absence or in the presence of task-irrelevant differences in level $\left(\Delta L_{\mathrm{i}}\right)$ or duration $\left(\Delta T_{\mathrm{i}}\right)$. For $1,000-\mathrm{Hz}$ tones, FDTs decrease with increasing duration up to $80-$ 

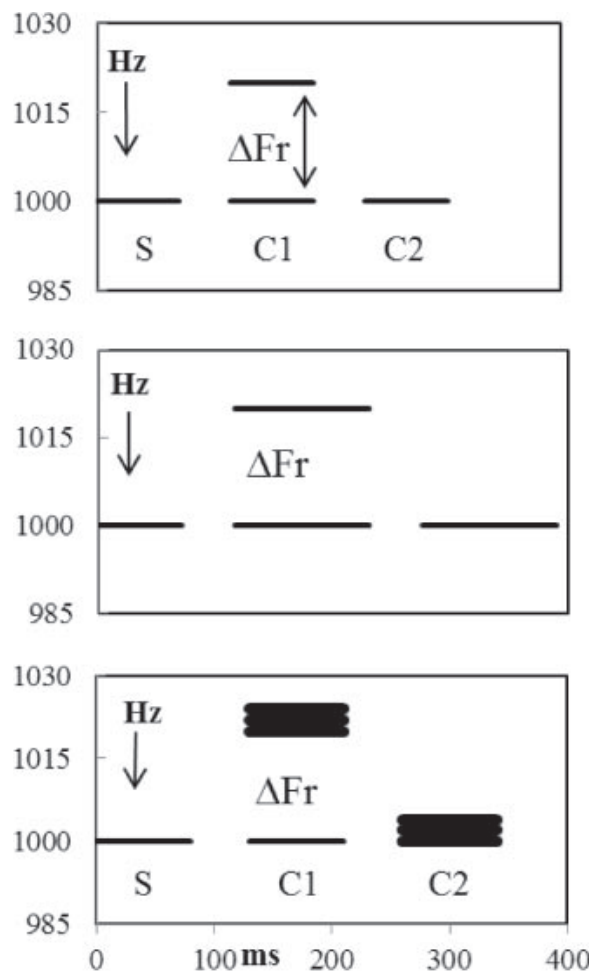

Fig. 1 Trial structure in the Control, $\Delta T_{\mathrm{i}}$, and $\Delta L_{\mathrm{i}}$ conditions. Horizontal line segments depict the targettone frequency (ordinate) and duration (abscissa). Each trial includes a standard (S) followed by two comparison $(\mathrm{C} 1$ and $\mathrm{C} 2)$ tones, respectively, in the 1st, 2nd and 3rd observation interval. Control condition (top): $\mathrm{C} 1$ includes a task-relevant frequency difference $\left(\Delta F_{\mathrm{r}}\right)$ but no irrelevant differences. Irrelevant duration (center): $\mathrm{C} 1$ includes $\Delta F_{\mathrm{r}}$, and both $\mathrm{C} 1$ and $\mathrm{C} 2$ include $\Delta T_{\mathrm{i}}$ (long segments). Irrelevant level (bottom): $\mathrm{C} 1$ includes $\Delta F_{\mathrm{r}}$, and both $\mathrm{C} 1$ and $\mathrm{C} 2$ include $\Delta L_{\mathrm{i}}$ (thick segments); $\mathrm{C} 1$ includes a frequency increment relative to $\mathrm{S}$ and $\mathrm{C} 2$ and both comparisons are louder than $\mathrm{S}$; inter-observation intervals scaled to $1 / 10$ of $500 \mathrm{~ms}$.

$100 \mathrm{~ms}$ [9]; thus, the $80-\mathrm{ms}$ tone helped the elders to master the task. In the $3 \mathrm{I} / 2 \mathrm{AFC}$ task, each trial presented a standard (S) followed by two comparisons ( $\mathrm{C} 1$ and $\mathrm{C} 2)$, respectively, in the 1st, 2nd, and 3rd observation interval, separated by $500 \mathrm{~ms}$ of silence (see Fig. 1). A frequency increment $\left(\Delta F_{\mathrm{r}}\right)$ was added to one comparison, chosen randomly with equal probability, and participants had to indicate the interval (2nd or 3rd) that included $\Delta F_{\mathrm{r}}$; this control condition did not include task-irrelevant differences. In the irrelevant leveldifference $\left(\Delta L_{\mathrm{i}}\right)$ condition, $\Delta F_{\mathrm{r}}$ was added again to one comparison but both comparisons included also positive 7.0or $14.0-\mathrm{dB}$ irrelevant level differences $\left(\Delta L_{\mathrm{i}}\right)$; in all trials, the size of $\Delta L_{\mathrm{i}}$ was the same for both comparisons. In the irrelevant duration-difference $\left(\Delta T_{\mathrm{i}}\right)$ condition, both comparisons included also positive 50 - or $100-\mathrm{ms}$ duration differences $\left(\Delta T_{\mathrm{i}}\right)$. Figure 1 depicts the trial structure of the 3 conditions studied: control, $\Delta T_{\mathrm{i}}$ and $\Delta L_{\mathrm{i}}$; in terms of level or duration, both comparisons were different from $\mathrm{S}$, but in terms of frequency only one comparison was different from $\mathrm{S}$. To respond correctly, participants had to attend to $\Delta F_{\mathrm{r}}$ while attempting to ignore $\Delta L_{\mathrm{i}}$ or $\Delta T_{\mathrm{i}}[5,6]$. In the $3 \mathrm{I} / 2 \mathrm{AFC}$ task, the level or duration difference was only between the standard and the two comparisons, and the latter two were always equal in level or duration; participants could respond correctly by attending to the frequency difference between the two equallevel or equal-duration comparisons. Since both comparisons conveyed large irrelevant differences, they were salient to all participants. Within each trial block (i.e., adaptive staircase), the irrelevant difference dimension (level or duration) was held constant but the irrelevant-difference size ( 7 or $14 \mathrm{~dB} ; 50$ or $100 \mathrm{~ms}$ ) was sampled randomly with equal probability. The test order of the 3 conditions was counterbalanced to control for practice and fatigue effects.

2.3. Frequency discrimination thresholds (FDT)

On each trial, the size of $\Delta F_{\mathrm{r}}$ was specified by an adaptive rule [10] estimating FDTs at the $71 \%$-correct level. For young adults, the adaptive rule started with a $50-\mathrm{Hz} \Delta F_{\mathrm{r}}$ that decreased by $2.0 \mathrm{~Hz}$ after 2 consecutive correct responses; $\Delta F_{\mathrm{r}}$ increased by $2.0 \mathrm{~Hz}$ after an error or a correct response followed by an error. For older adults, the same rule was used but starting with a $70-\mathrm{Hz} \Delta F_{\mathrm{r}}$ to facilitate mastering the task. An FDT estimate was defined as the average of the frequency increments specified in the last five reversals of adaptive tracks comprising eight reversals; FDT estimates from 8 tracks were averaged to compute the FDT of the control, $\Delta L_{\mathrm{i}}$, and $\Delta T_{\mathrm{i}}$ condition. With each participant, a total of 24 adaptive tracks ( 8 per condition) were collected on a single $1.0-1.5 \mathrm{~h}$ test session, breaks included. To take into account accuracy differences between young and older adults in the control condition, the age groups were matched in terms of training amount or number of adaptive tracks, and the effects of $\Delta T_{\mathrm{i}}$ or $\Delta L_{\mathrm{i}}$ were assessed against the control FDT of each group.

\subsection{Participants}

The older adults included ten 64-74 years old (mean = 70.3), healthy participants (9 female); 8 normal hearing and 2 mild-to-moderate, high-frequency hearing impaired, but not exceeding the $1,000-\mathrm{Hz}$ norm by $\geq 30 \mathrm{~dB}$. Thus, in all participants the $75-\mathrm{dB}, 80-\mathrm{ms}, 1,000-\mathrm{Hz}$ target tone was $\geq 35 \mathrm{~dB}$ above detection threshold and clearly audible. All older adults scored $\geq 90 \%$ correct in a mental-status questionnaire [11], ruling out cognitive deficits. The young-adult group comprised ten normal-hearing graduate students, 22-31 years old (mean $=24.5 ; 6$ female). The OUHSC Institutional Review Board approved the study protocol; participants consented in writing and were paid for listening.

\section{Results}

For both age groups, Fig. 2 shows the mean FDTs measured without (control) and with task-irrelevant differences in duration $\left(\Delta T_{\mathrm{i}}\right)$ or level $\left(\Delta L_{\mathrm{i}}\right)$. Because our goal was comparing effects of irrelevant stimulus differences in young and older participants matched in terms of training amounts, the FDTs do not represent best-possible differential sensitivity; this would require far more training in older than in young adults [6] and confound the aging effect. Without irrelevant differences, the control FDTs of both age groups are larger than those measured in highly-trained participants [12], and the elder's FDTs far exceed those of the young adults in all 


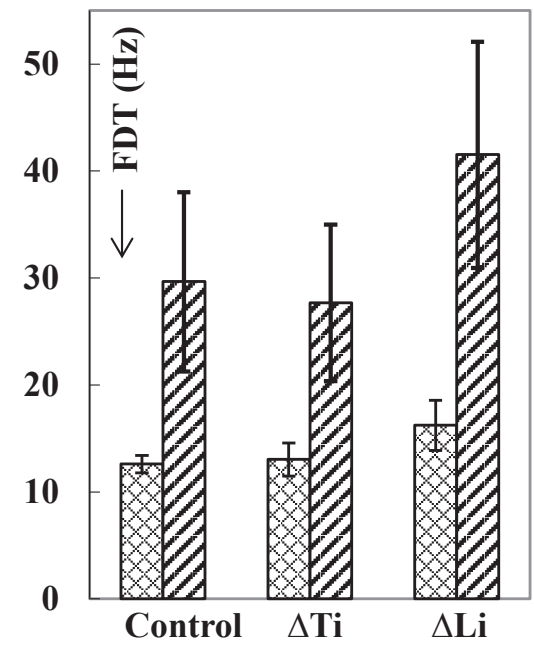

Fig. 2 Columns show the mean FDT of young (crisscross) and older (slant) adults in the control, irrelevantduration $\left(\Delta T_{\mathrm{i}}\right)$, and irrelevant-level $\left(\Delta L_{\mathrm{i}}\right)$ difference conditions. Error bar is the standard error of the mean.

three conditions. Thus, within each age group, the irrelevantdifference effects were assessed in relation to the control FDTs. In both groups, FDTs measured in the midst of $\Delta T_{\mathrm{i}}$ are nearly equal to those of the control FDTs; although it was large, $\Delta T_{\mathrm{i}}$ was easily ignored by all participants. However, in the presence of $\Delta L_{\mathrm{i}}$ the mean FDTs are clearly larger than the control FDTs, especially in older adults.

The significance of the FDT disparities across age groups and across irrelevant-difference conditions (control, $\Delta L_{i}$, and $\Delta T_{\mathrm{i}}$ ) was assessed by a fixed-effects ANOVA with age as between-group factor (2 layers, this term avoids confusion with "dB level") and irrelevant-difference as a within-group factor (3 layers). The ANOVA revealed significant main effects of age $[F(1,18)=4.73, \mathrm{MS}=5,415, \quad p=0.04]$, irrelevant-difference condition $[F(2,36)=11.61, \quad \mathrm{MS}=$ 443.3, $p<0.001$ ], and a significant interaction between age and irrelevant difference $[F(2,36)=4.1, \quad \mathrm{MS}=156.70$, $p<0.025]$. Across age groups, pairwise tests (Student's $t$-test, $p \leq 0.05$ ) showed significantly larger FDTs in older than in young adults in the control $(p \leq 0.03$, one-tail), irrelevant-duration ( $p \leq 0.03$, one-tail), and irrelevant-level ( $p \leq 0.03$, two-tail) conditions. As expected, in all conditions the elder's FDTs were larger than the young adult's (thus validating the one-tail significance levels); elders underperform even without irrelevant differences. Within the young group, in the presence of $\Delta L_{\mathrm{i}}$, the mean FDT was not significantly larger than the control FDT ( $p \leq 0.09$, two-tail), but was significantly larger than the mean FDT in the midst of $\Delta T_{\mathrm{i}}(p \leq 0.01$, two-tail). Within the older group, in the presence of $\Delta L_{\mathrm{i}}$, the mean FDT was significantly larger than both the control FDT ( $p \leq 0.02$, two-tail) and the FDT in the midst of $\Delta T_{\mathrm{i}}$ ( $p \leq 0.01$, two-tail); the latter FDT was not significantly larger than the control FDT ( $p=0.23$, two-tail). That is, the elders easily ignored $\Delta T_{\mathrm{i}}$, but had considerable difficulty ignoring $\Delta L_{\mathrm{i}}$. This is consistent with inability to ignore irrelevant information in older adults; however, this deficit is not observed amid $\Delta T_{\mathrm{i}}$. In addition, the standard error of the means are much larger in older than in young adults, pointing to wider FDT variability in the elders.

\section{Discussion and conclusions}

In older but not in young adults, our results show that the ability to attend to and discern task-relevant frequency differences $\left(\Delta F_{\mathrm{r}}\right)$ is degraded by the simultaneous presentation of task-irrelevant level differences $\left(\Delta L_{\mathrm{i}}\right)$. This degradation inflated the FDTs, relative to those measured without $\Delta L_{\mathrm{i}}$; however, attention deficits did not obtain with simultaneous presentation of irrelevant-duration differences $\left(\Delta T_{\mathrm{i}}\right)$. Thus, rather than generalized, the degradation seems to be specific to the particular combination of relevant- and irrelevant-difference dimensions. Attention deficits were observed in elders with normal hearing and cognitive ability, and with adequate training in the irrelevant-difference tasks. Since peripheral-receptor injury cannot account for the elders' attention deficit, their difficulty ignoring irrelevant differences must be linked to central-processing constraints; to account for this, two possible mechanisms are described below.

Strength of pitch-loudness interactions. Ignoring the $\Delta L_{\mathrm{i}}$ in frequency discrimination tasks could be difficult partly because loudness and pitch interact with one another, and this interaction could be stronger in older than in young adults; that is, their ability to separate these dimensions at the perceptual level would be reduced compared to that of young adults. It has been proposed [7,8] that frequency discrimination is based on detecting changes in excitation level along the low-frequency slope of the excitation pattern, and that the FDT corresponds to a $1-\mathrm{dB}$ change along this slope. Thus, in the present tasks, the positive $7-14 \mathrm{~dB} \Delta L_{\mathrm{i}}$ would tend obscure the much smaller excitation-level changes associated with relevant frequency differences, thereby increasing the FDT. In frequency discrimination of tones lower than $2.0 \mathrm{kHz}$, an increase in level tends to cancel out the pitch shift induced by a positive frequency increment, but a decrease in level tends to enhance the pitch shift [13]; that is, the irrelevantlevel differences may lessen the perceptual effect of positive task-relevant frequency differences. In pitch-classification tasks [5], young adults perceive high-pitched, loud sounds as being more congruent than high-pitched, soft sounds; that is, pitch increments are perceived as been more similar to an increase than to a decrease in loudness.

Inflexible perceptual strategy. Without irrelevant differences, each trial presented three tones and participants could discern $\Delta F_{\mathrm{r}}$ based on comparing, within trial, the memory traces of the standard and the two comparison tones. This strategy would work well in the midst of $\Delta T_{\mathrm{i}}$ because it did not alter the frequency and pitch relations between $S$ and $\mathrm{C} 1, \mathrm{C} 2$; in the midst of $\Delta T_{\mathrm{i}}$, perceiving them as a single group and relying on within-trial comparisons would not hinder discrimination accuracy. If anything, by lengthening the $\mathrm{C} 1$ and $\mathrm{C} 2$ duration, $\Delta T_{\mathrm{i}}$ would improve discrimination (i.e., lower FDTs) because the task-relevant frequency difference lasted 50-100 ms longer than in the control condition. In fact, in the midst of $\Delta T_{i}$, the elder's FDTs were slightly but not significantly lower than in the control condition. In addition, lengthening the $\mathrm{C} 1$ and $\mathrm{C} 2$ duration slows down the presentation rate, which can also facilitate $\Delta F_{\mathrm{r}}$ discrimina- 
tion, especially in older adults. In contrast to the $\Delta T_{\mathrm{i}}$, the $\Delta L_{\mathrm{i}}$ alter both the level and the pitch of $\mathrm{C} 1$ and $\mathrm{C} 2$; thus, the standard tone may no longer function as a reference that is effective to discern $\Delta F_{\mathrm{r}}$ in one of the comparisons. In this case, the best strategy would be to ignore the standard and focus attention on $\mathrm{C} 1$ and $\mathrm{C} 2$; that is, to separate perceptually the standard from $\mathrm{C} 1$ and $\mathrm{C} 2$ and decide which of these two includes $\Delta F_{\mathrm{r}}$. Frequency discrimination between comparisons would be more accurate than between the standard and the comparisons. However, accomplishing this perceptual segregation is difficult because the tones are of short duration, similar in frequency, and close in time. In addition, because the $\Delta L_{\mathrm{i}}$ are large and salient, they tend to draw attention away from the relevant frequency difference. Performing this perceptual segregation could be more difficult for older that young adults, thereby limiting their ability to ignore $\Delta L_{\mathrm{i}}$ and increasing the FDTs. The basic mechanism would be limited cognitive flexibility that prevents elders from re-orienting attention [14].

\section{Acknowledgments}

Research funded by Allied Health, OUHSC and the ABMRF (B. Espinoza-Varas, PI).

\section{References}

[1] P. A. Tun and A. Wingfield, "One voice too many: Adult age differences in language processing with different types of distracting sounds," J. Gerontol. B. Psychol. Sci., 54B, P317P327 (1999).

[2] C. Lustig, L. Hasher and R. T. Zacks, "Inhibitory deficit theory: Recent developments in a 'new view,' in Inhibition in Cognition, D. S. Gorfein and C. M. MacLeod, Eds. (American Psychological Association, Washington, DC, 2007), pp. 145-162.
[3] W. Ellermeier and K. Zimmer, "The psychoacoustics of the irrelevant sound effect," Acoust. Sci. \& Tech., 35, 10-16 (2014).

[4] S. Gordon-Salant and P. J. Fitzgibbons, "Selective cognitive factors and speech recognition performance among young and elderly listeners," J. Speech Lang. Hear. Res., 40, 423-431 (1997).

[5] B. Espinoza-Varas and H. Jang, "Selective attention to pitch amid conflicting auditory information," Psychol. Res., 75, 159-178 (2011).

[6] B. Espinoza-Varas and H. Jang, "Backward-masked frequency discrimination by young and older adults: Effects of masker level variability," Acta Acust. united Acu., 92, 457-467 (2006).

[7] G. B. Henning, "Frequency discrimination of random-amplitude tones," J. Acoust. Soc. Am., 39, 336-339 (1966).

[8] D. S. Emmerich, W. Ellermeier and B. Butensky, "A reexamination of the frequency discrimination of randomamplitude tones, and a test of Henning's modified energydetector model," J. Acoust. Soc. Am., 85, 1653-1659 (1989).

[9] R. L. Freyman and D. A. Nelson, "Frequency discrimination as a function of tonal duration and excitation-pattern slopes in normal and hearing-impaired listeners," J. Acoust. Soc. Am., 79, 1034-1044 (1986).

[10] H. Levitt, "Transformed up-down methods in psychoacoustics,” J. Acoust. Soc. Am., 49, 467-477 (1971).

[11] E. Pfeiffer, "A short portable mental status questionnaire for the assessment of organic brain deficit in elderly patients," J. Am. Geriat. Soc., 23, 433-441 (1975).

[12] C. C. Wier, W. Jesteadt and D. M. Green, "Frequency discrimination as a function of frequency and sensation level," J. Acoust. Soc. Am., 81, 178-182 (1977).

[13] T. D. Rossing and A. J. Houtsma, "Effects of signal envelope on the pitch of short sinusoidal tones," J. Acoust. Soc. Am., 79, 1926-1933 (1986).

[14] R. Naatanen, Attention and Brain Function (Erlbaum, Hillsdale, N.J., 1992). 